\title{
A female with systemic lupus erythematosus and Streptococcal pneumonia treated with intravenous immunoglobulins (IVIG).
}

Angel Justiz-Vaillant ${ }^{1 *}$ Belkis Ferrer Cosme ${ }^{2,3}$ Niurka Ramírez Hernández $z^{4,5}$

${ }^{1}$ Department of Para-clinical Sciences. Faculty of Medical Sciences. The University of the West Indies. St. Augustine. Trinidad and Tobago. West Indies. ${ }^{2}$ Higher Institute of Medical Sciences of Santiago de Cuba.

${ }^{3}$ Saturnino Lora Clinical and Surgical Provincial Teaching Hospital. Santiago de Cuba.

${ }^{4}$ Havana University of Medical Sciences.

${ }^{5}$ Freyre de Andrade General Teaching Hospital. Havana City. Cuba.

*Corresponding author: angel.vaillant@ sta.uwi.edu

\begin{abstract}
The systemic lupus erythematosus (SLE) is a complex autoimmune disorder with a wide array of clinical manifestations, including chest infections. S. aureus is the most common bacteria that cause infectious pneumonia in SLE. Other bacteria, such as S. pneumoniae has been implicated as a cause of lung infection. In this study we aimed to use intravenous immunoglobulins (IVIG) as only immunotherapy for the management of a SLE in a Hispanic woman. Laboratory investigations for the diagnosis of SLE were done including the detection of anti-nuclear antibodies (ANA) and SLE confirmation by the detection of high titers of anti-dsDNA antibodies. Serum levels of $\mathrm{C} 3$ and $\mathrm{C} 4$ were assessed as well as quantification of immunoglobulins. The SLEDAI score was measured to determine whether a significant degree of disease activity existed and as prognostic value. The evaluation of the chest infection was performed by chest-X-ray at the beginning and after treatment. She was treated with $10 \mathrm{~g} / \mathrm{day}$ of IVIG for six consecutive days and thereafter $10 \mathrm{~g} /$ monthly. The immunological evaluation demonstrated that this patient presented with a flare of SLE during a pneumococcal pneumonia. Hight titers of ANA and anti-dsDNA antibodies were detected as well as low C3 and C4, and elevated levels of immunoglobulins. The SLEDAI score fall from 10 to below 3 and the chest infection cleared up. IVIG treatment was effective in the treatment of a pneumonia associated SLE crisis. IVIG demonstrated once more that can be used as immunosuppressor, immunomodulator, and anti-microbial in patients with SLE, avoiding the use of steroids.
\end{abstract}

Keywords: Systemic lupus erythematosus (SLE), Intravenous immunoglobulins (IVIG), Autoantibody, Autoimmune disease, Pneumonia. 


\section{Introduction}

Certain bacteria, including the Streptococci, can cause pneumonia in immunocompromised individuals as SLE patients. Streptococci are gram-positive spherical bacteria that present in two forms: pairs or chains during growth. They are part of the normal human flora and distributed widely in nature and associated with increased morbidity and mortality [1].

The systemic lupus erythematosus (SLE) is a complex autoimmune disorder. It develops in genetically prone patients, where the influence of various environmental factors causes the development of many autoantibodies. It is a common autoimmune disease. A wide array of clinical manifestations characterizes it. The most common signs of illness are photosensitivity, oralulcers, pleuritis, pneumonia, pericarditis, arthritis, kidney problems, blood cell abnormalities, seizures, and psychosis. Antibodies in SLE react with critical components of the cell nucleus $[2,3]$.

SLE is mainly a condition of young women; it may occur from infancy to old age. It peaks between the ages of 15-40. Females are mostly affected (6-10:1), and blacks (and possibly Hispanics, Asians, and Native Americans) are affected more than whites [2,3].

The SLE prevalence varies worldwide and ranges as high as 1,500,000 cases, as reported by the Lupus Foundation of America [4]. A recent study estimated a 2005 incidence of 161,000 with definite SLE and 322,000 with definite or probable SLE [5].

IVIG is a product prepared from fractioning pools of thousands of plasma donations and collected at blood transfusion services [6-9]. Other proteins, such as traces of $\operatorname{IgM}$ and $\operatorname{IgA~[10],~cytokines,~}$ and immunomodulating peptides [11], can be in the preparation along with purified IgG. This paper described a compelling case of a young female with pneumonia and systemic manifestations of SLE and treated with IVIG produced at "Serum and Blood-Derivative Products Company "Adalberto Pesant".

\section{Case presentation}

This is the case of a woman with a well-known SLE that has previously reported [12]. A 23-year- 
old white female was sent to the Freyre de Andrade Hospital, Havana City, Cuba, with symptoms of malaise. She was well until a week ago, when she developed polyarthralgia, skin manifestations, and complaints of a chest infection including productive cough with yellow mucus, shortness of breath, and stabbing chest pain [12]. Since the age of 16 she was diagnosed SLE and since that age she has been admitted various times for evaluation and treatment.

Vital signs recorded the presence of fever $\left(101^{\circ} \mathrm{F}\right)$, oxygen saturation of $97 \%$ at room air, heart rate of 105 beats/min, respiratory rate of 22 breaths/min. Pulmonary examination revealed the presence of crackles, wheezing, and decreased breath sounds. Complete blood cell count showed the presence of leukopenia $\left(2600 / \mathrm{mm}^{3}\right)$. Hemoglobin of $9.8 \mathrm{~g} / \mathrm{dL}$ and erythrocyte sedimentation rate of $68 \mathrm{~mm} / \mathrm{h}$.

Antinuclear antibodies (ANA) were present at a titer of 1/2000 with a homogeneous pattern. Laboratory findings include proteinuria, raised gammaglobulin fraction on the serum protein electrophoresis, and C-reactive protein (CRP) of $165 \mathrm{mg} / \mathrm{L}$. Besides, anti-dsDNA antibodies were found to be in a concentration of $98 \mathrm{IU} / \mathrm{ml}$, along with low levels of C3 and C4. HIV, $\mathrm{HBV}$, and HCV serologies were negative. Chest $\mathrm{X}$ - rays revealed features of pleural effusion, and lobal consolidation in the right lungs, which was consistent with lobal pneumonia. In sputum and blood culture was isolated Streptococcus pneumoniae using standard microbiological techniques.

This woman was a well-known SLE case that explains why she was managed in the past with non-steroid anti-inflammatory drugs, IVIG, corticosteroids, and immune suppressants. Her physical examination showed that she had arterial hypertension $(150 / 100 \mathrm{mmHg})$. She had the classic "butterfly rash" and other signs of vasculitis, in addition, signs of pericardial friction rub, and the echocardiography confirmed a moderate-sized pericardial effusion.

The patient was sent to the intensive care unit (ICU), where she was treated with $10 \mathrm{~g} / \mathrm{day}$ of IVIG for six consecutive days. There was a marked clinical improvement of the pulmonary manifestations after completion of the immunotherapy with IVIG. Also, there was an improvement in the skin and cardiovascular problems.

Laboratory findings showed an increase in the activity of the hemolytic complement, lower titers of anti-dsDNA, and anti-red blood cell antibodies. A chest X-ray showed a resolution of pleurisy and the lobal pneumonia. The patient was carried to the Internal Medicine ward, where 
she was followed for five days, and then she was sent home.

Furthermore, she was treated with IVIG (10 g/monthly). Clinically and humoral remission was achieved for a10 months, where she did not complain about other signs and symptoms of chest infection nor other SLE manifestations. The SLEDAI score was assessed to evaluate the clinical status and showed a score of 12 and $<3$ before and after the treatment with IVIG, respectively. She remains on remission with few altered serological parameters.

\section{Discussion}

The use of purified IgG for the treatment of both the SLE crisis and pneumonia caused by pneumococci is described. Mostly the lung infection seen in SLE is due to Staphylococci and is treated with antibiotics. Here we demonstrated that both SLE and bacterial pneumonia could be treated with IVIG only, without the need of steroids or immunosuppressors due to the IVIG's immunosuppressive effects and the presence of a wide range of anti-bacterial antibodies.

A dosage of 5-10 g/day of IVIG was used for six consecutive days during the SLE crisis, and then $10 \mathrm{~g}$ monthly as a maintenance dose. A reduction of the SLEDAI score proved the beneficial effects of purified IgG therapy in this case.

IVIG was useful for the clearance of pneumonia and other manifestations of lupus.

This case needed a higher immunoglobulin dose because she experienced an acute respiratory infection in addition to vasculitis [8]. Also, IVIG therapy was enough for the control of possibly other subclinical infections associated with SLE as antibodies directed against a broad spectrum of microorganisms, including bacteria, can be present in high levels in the IVIG [9].

SLE patients are immunodeficient due to the nature of the disease and the immunotherapy used to suppress the immune system. Recurrent chest infections may be a late clinical manifestation of this autoimmune disease. Garcia-Guevara and collaborators (2018) reported that pneumonia remains the leading cause of mortality in patients with systemic lupus erythematosus (SLE), and the most common bacteria isolated are S. aureus, but other genera of bacteria have been cited [10-11].

In this study, S. pneumoniae was demonstrated in blood culture and presented not only as a cause of pneumonia but bacteremia. The pulmonary systemic lupus erythematosus (SLE) may manifest 
by lupus pneumonitis that can mimic acute infectious pneumonia in all aspects (clinically and radiographically). Both present with fever, dyspnea, and chest pain. Nevertheless, an infectious microorganism can be isolated at the latest. IVIG has an antimicrobial effect against viruses and bacteria $[8,12]$ through a mechanism of neutralization and opsonization. Besides, it has a positive effect on activated B cells [12] that contribute to the production of specific antibodies against bacterial antigens.

'Declarations of interest: none.'

Ethical approval: It was obtained by the Ethics Committee of the Freyre de Andrade General Teaching Hospital. Informed consent was obtained from the patient for being included in the study. Acknowledgment: To Dr. Niurka Ramirez for her invaluable input in the management of this patient.

\section{References}

1. H. Yumoto, K. Hirota, K. Hirao, M. Ninomiya, K. Murakami, H. Fujii, Y. Miyake. The Pathogenic Factors from Oral Streptococci for Systemic Diseases. Int J. Mol. Sci. 20 (2019) doi: 10.3390/ijms20184571.

2. Pisetsky, D.S., Buyon, J.P. and Manzi, S., Chapter 17. "Systemic lupus erythematosus”. In: J.H. Klippel, L.J. Crofford, J.H. Stone, \& C.M. Weyand.

Primer on the Rheumatic Diseases. Edition 12. Arthritis Foundation, Atlanta, GA., 2001.

3. V. Rus, A. Hajeer, \& M.C. Hochberg. Chapter 7. "Systemic lupus erythematosus". In: A.J. Silman, \& M.C. Hochberg (eds). Epidemiology of the Rheumatic Disease. 2nd edition. Oxford University Press, New York, 2001.

4. R.C. Lawrence, D.T. Felson, C.G. Helmick, L.M. Arnold, H. Choi, R.A. Deyo, S. Gabriel, et al. For the National Arthritis Data Workgroup. "Estimates of the prevalence of arthritis and other rheumatic conditions in the United States: Part II”, Arthritis. Rheum. 58 (2008) 26-35. doi: 10.1002/art.23176.

5. C.G. Helmick, D.T. Felson, R.C. Lawrence, S. Gabriel, R. Hirsch, C.K. Kwoh, M.H. Liang, et 
al. For the National Arthritis Data Workgroup. "Estimates of the prevalence of arthritis and other rheumatic conditions in the United States: Part I", Arthritis. Rheum. 58 (2008) 15-25. doi: 10.1002/art.23177.

6. J. Bayry J, V.S. Negi, S.V. Kaveri. Intravenous immunoglobulin therapy in rheumatic diseases. Nat. Rev. Rheumatol. 7 (2011) 349-59. doi: 10.1038/nrrheum.2011.61.

7. P.R. Timmerman. Intravenous immunoglobulin in oncology nursing practice. Oncol. Nurs. Forum. 20 (1993) 69-75.

8. R. Lemieux, R. Bazin, S. Néron. Therapeutic intravenous immunoglobulins. Mol. Immunol. 42 (2005) 839-48. doi: 10.1016/j.molimm.2004.07.046

9. T. Sapir, Y. Shoenfeld. Facing the enigma of immunomodulatory effects of intravenous immunoglobulin. Clin. Rev. Allergy. Immunol. 29 (2005) 185- 99. doi: 10.1385/CRIAI:29:3:185.

10.G. García-Guevara, R. Ríos-Corzo, A. Díaz-Mora, M. López-López, J. Hernández-Flores, H. Fragoso-Loyo, J. Ávila-Vázquez, et al. Pneumonia in patients with systemic lupus erythematosus: Epidemiology, microbiology and outcomes. Lupus. 27 (2018) 1953-1959. doi: 10.1177/0961203318799207.

11.Enomoto N, Egashira R, Tabata K, Hashisako M, Kitani M, Waseda Y, T. Ishizuka, et al. Analysis of systemic lupus erythematosus-related interstitial pneumonia: a retrospective multicentre study. Sci Rep. 2019 May 14;9(1):7355. doi: 10.1038/s41598-019-43782-7.

12. Vaillant AAJ, Ramirez N, Cadiz A, Akpaka PE (2013) Case Reports on the Use of Intravenous Immunoglobulins (IVIG) in the Treatment of Systemic Lupus Erythematosus (SLE). J Clin Case Rep 3: 263. doi:10.4172/2165-7920.1000263 